\section{Validade científica de conhecimento epidemiológico gerado com base no estudo Saúde Bucal Brasil 2003}

\author{
Scientific validity of epidemiological knowledge \\ based on data from the Brazilian Oral Health \\ Survey (SB Brazil 2003)
}

\author{
${ }^{1}$ Faculdade de Saúde \\ Pública, Universidade de \\ São Paulo, São Paulo, Brasil. \\ 2 Escola de Artes, Ciências e \\ Humanidades, Universidade \\ de São Paulo, São Paulo, \\ Brasil. \\ 3 Centro de Ciências \\ Biológicas e da Saúde, \\ Pontifícia Universidade \\ Católica do Paraná, Curitiba, \\ Brasil. \\ 4 Programa de Pós-graduação \\ em Saúde Pública \\ Universidade Federal \\ de Santa Catarina, \\ Florianópolis, Brasil. \\ ${ }^{5}$ Faculdade de Odontologia \\ de Piracicaba, Universidade \\ Estadual de Campinas, \\ Piracicaba, Brasil. \\ 6 Departamento de \\ Odontologia, Universidade \\ Federal do Rio Grande do \\ Norte, Natal, Brasil. \\ Correspondência \\ P. C. Narvai \\ Faculdade de Saúde Pública, \\ Universidade de São Paulo. \\ Av. Dr. Arnaldo 715, \\ São Paulo, SP \\ 01246-904, Brasil. \\ pcnarvai@usp.br
}

\begin{abstract}
Indicators and analyses that used the database from SB Brazil 2003 (the most recent nationwide oral health survey) have been criticized as unreliable due to sampling problems. The current study countered that this critique was based solely on statistical concepts, unsupported by empirical evidence. The critique's essentially epistemic approach leads to peremptory reductionism that denies other forms of knowledge and fails to recognize the multidisciplinary nature of epidemiology. The current study retrieves information on the implementation of the oral health survey and its impact on knowledge output in the field. The article draws an analogy between science and art, demonstrating the multifaceted images obtained by both. Thus, recognition of validity requires a full grasp of the field and appropriate use of value criteria. The current article concludes that use of the SB Brazil 2003 database is a reliable and relevant application of epidemiology to oral health.
\end{abstract}

Uses of Epidemiology; Dental Health Surveys; Oral Health
Paulo Capel Narvai 1

José Leopoldo Ferreira Antunes 2

Samuel Jorge Moysés 3

Paulo Frazão 1

Marco Aurélio Peres 4

Karen Glazer Peres 4

Maria da Luz Rosário de Sousa 5

Angelo Giuseppe Roncalli 6

\section{Introdução}

Em seu primeiro número de 2009, Cadernos de Saúde Pública publicou um artigo ${ }^{1}$ que afirma não serem válidas as estimativas sobre condições de saúde bucal da população brasileira realizadas com base no banco de dados gerado pela pesquisa Condições de Saúde Bucal da População Brasileira 2002-2003, conhecida como SB Brasil 2003. Para os autores do artigo, o Ministério da Saúde "talvez tenha induzido o uso dos dados do SB Brasil 2003 por diversos pesquisadores, que publicaram artigos científicos em revistas nacionais e internacionais, sem perceberem que os resultados não eram inferências estatisticamente válidas para os grupos etários da população de pesquisa (brasileira ou por macrorregião) porque eram referidos e limitados ao grupo estudado (estatísticas da amostra)” 1 (p. 48). Essa afirmação categórica representa importante restrição sobre todos os trabalhos que até agora analisaram essas condições baseando-se no banco de dados da pesquisa, e produz efeito inibidor sobre estudos em desenvolvimento. Além disso, os autores consideram que os levantamentos de saúde bucal realizados até o momento não produziram um quadro abrangente sobre a situação do Brasil e que, em conseqüência, pesquisadores e gestores não podem analisar essas condições nem definir metas programáticas.

Essas restrições não são infundadas e devem ser cuidadosamente consideradas pelos profis- 
sionais de saúde pública, gestores e pesquisadores. Nesse sentido, procurou-se examinar criticamente o texto em pauta, para estabelecer essa interlocução. O objetivo deste estudo foi contribuir para a reflexão sobre a validade dos dados do SB Brasil 2003, contextualizando, refletindo e interpretando as limitações estatísticas apontadas e quais são suas consequências para a validade dos estudos que utilizaram essa base de dados.

\section{Procedimentos metodológicos}

Foi analisado o artigo científico Pesquisa sobre as Condições de Saúde Bucal da População Brasileira (SB Brasil 2003): Seus Dados Não Produzem Estimativas Populacionais, mas Há Possibilidade de Correção, publicado em Cadernos de Saúde Pública 1. Os objetivos do artigo foram "demonstrar que o procedimento de amostragem do SB Brasil 2003 não foi concluído" 1 (p. 49) e alertar seus potenciais usuários que as "estimativas produzidas não são representativas da população brasileira". Além disso, admite haver possibilidades de "correção dos problemas identificados".

O "SB Brasil 2003" a que alude o artigo é a expressão utilizada para designar o levantamento epidemiológico intitulado Condições de Saúde Bucal da População Brasileira 2002-2003, realizado pelo Ministério da Saúde, com o objetivo de "produzir informações sobre as condições de saúde bucal da população brasileira e subsidiar o planejamentolavaliação de ações nessa área nos diferentes níveis de gestão do Sistema Único de Saúde, por meio da coordenação de um amplo esforço nacional para o estudo dessas condições e para criação e manutenção de uma base de dados eletrônica relativa aos principais problemas nesse campo, articulando diferentes agências e unidades de saúde, contribuindo na perspectiva da estruturação de um sistema nacional de vigilância epidemiológica em saúde bucal" 2 (p. 11).

Foi realizada análise documental utilizando-se técnicas de análise temática ${ }^{3}$. Os temas foram tratados mediante operações de desmembramento do texto em unidades, visando a identificar os diferentes núcleos de sentido, seguindo-se a seleção de categorias para fins de interpretação. As categorias selecionadas foram: validade, inferência, representatividade, estimativa, enviesamento, precisão, planejamento, gestão e vigilância.

Os núcleos de sentido identificados foram contextualizados mediante a apresentação de informações sobre a realização anterior de levantamentos dessa natureza no país. Foram contempladas informações sobre o processo que culminou na realização do SB Brasil 2003 e suas conse- quências, em termos da utilização de seus dados na produção de conhecimento de interesse para o planejamento e gestão dos serviços de saúde. Por fim, foram realizados ensaios de ponderação estatística dos dados desse levantamento, e foi proposta uma analogia das imagens obtidas pelo conhecimento científico, com imagens produzidas pelo conhecimento artístico, argumentando-se que, em ambas as situações, os saberes gerados a partir do objeto cognoscível assumem feições variadas e, portanto, o reconhecimento de sua validade requer amplo domínio do objeto e operar com adequados critérios de valor.

\section{Aspectos éticos}

Todos os autores deste artigo conduziram estudos em que o banco de dados do SB Brasil 2003 foi utilizado. A.G.R. e P.F. participaram do planejamento e execução da pesquisa que gerou o banco de dados. Assim, registramos a possibilidade de conflito de interesses entre essas atividades e as considerações apresentadas neste artigo.

\section{Resultados e discussão}

Do conjunto de objeções à pesquisa SB Brasil 2003 emergiram as seguintes idéias centrais: (1) levantamentos nacionais de saúde bucal anteriores "não foram suficientes para a construção de um quadro abrangente sobre as condições de saúde bucal da população brasileira" 1 (p. 48); (2) o plano amostral seria falho e inconcluso, " $a$ falta dos pesos amostrais inviabiliza a estimação de totais populacionais" 1 (p. 49); (3) os resultados do SB Brasil 2003 não permitem inferências válidas, as "estimativas produzidas não são representativas da população brasileira” 1 (p. 49); e (4) a ausência de "inferências válidas para a população" impediria que "pesquisadores e gestores possam analisar as reais [sic] condições de saúde bucal no país e traçar metas para seu melhoramento" 1 (p. 57).

\section{Levantamentos de saúde bucal}

A despeito das reconhecidas limitações dos levantamentos nacionais de saúde bucal de 19864 e de 1996 5, questiona-se: (a) Seria sensato firmar posição absoluta, com base apenas em critérios estatísticos, sem levar em conta outras racionalidades ou informações de outras disciplinas que fundamentam a epidemiologia, e afirmar que tais inquéritos " $n \tilde{a} o$ eram representativos da população das regiões e, muito menos, da população brasileira"? 1 (p. 48); (b) Representatividade estatística esgotaria a capacidade de representação 
de estudos epidemiológicos? O artigo sob análise se auto-referencia e sustenta no solo estatístico, somente. Sendo auto-referencial só encontra sentido pleno na cientificidade em que se apóia. É epistemocêntrico em seus próprios termos e não admite outras concepções sobre "representatividade" em ciência. Recusa outras formas cientificamente "válidas" de representação da realidade, sobretudo na epidemiologia que é campo essencialmente multidisciplinar, não meramente matemático. Bastaria mencionar a contribuição de diferentes correntes de pensamento sobre representação social, tema tão caro às ciências sociais que também são fundantes da epidemiologia 6 . A complexa questão da "validade" da representação de população em estudos epidemiológicos restou reduzida, superdimensionando-se o significado de pesos amostrais e variâncias em relação a todas as dimensões envolvidas; (c) Por não se poder afirmar que certos valores correspondem a estimativas representativas da população, é possível deduzir que estes valores, necessariamente, não são válidos?; (d) É apropriado afirmar, imperativamente, que dados de inquéritos populacionais não são representativos, portanto não têm validade, refutando-os sem apresentar base empírica que demonstre sua não-validade? O artigo não apresentou provas empíricas de que as estimativas produzidas seriam inválidas. Apenas especulou essa possibilidade com base teórica em pressupostos da amostragem probabilística. Pode-se deduzir que as estimativas não são representativas por não ter havido a ponderação da amostra?

\section{Plano amostral e coleta de dados}

\section{do SB Brasil 2003}

Uma característica importante do SB Brasil 2003 foi sua articulação a uma estratégia mais ampla de construção do sistema de vigilância em saúde bucal. Outro aspecto relevante foi o esforço de difundir instrumentos e desenvolver pessoal vinculado ao SUS para impulsionar a prática epidemiológica na gestão dos serviços de saúde. As experiências anteriores de estudos nacionais foram centralizadas e os pontos de coleta de dados limitados, não criando oportunidades para envolver os diferentes setores (universidades, serviços, entidades, associações) e instâncias de níveis municipal e estadual.

"Pela primeira vez na história das políticas públicas em saúde bucal no Brasil um projeto desta envergadura surge como resultado de uma produção coletiva e de um processo democrático de discussão, fato que só foi possivel em virtude do interesse e do empenho de todos que dele participaram". Essa afirmação consta das Considera- ções Finais do Relatório do Processo de Discussão Pública do Anteprojeto SB2000. Esse anteprojeto foi encaminhado para as secretarias estaduais de saúde, instituições de ensino superior e entidades odontológicas. Foi criado um sítio eletrônico na rede mundial de computadores para livre acesso de informações e envio de contribuições. De setembro de 1999 a março de 2000 foram recebidas vinte propostas. Todas foram analisadas, a maioria foi incorporada ao projeto final, e o comitê técnico de coordenação respondeu a cada proposição. Em abril de 2000, foi disponibilizado o relatório com as propostas e comentários.

O anteprojeto do estudo também foi objeto de mesas de debates e fóruns de discussão em congressos e eventos técnico-científicos de abrangências local, regional e nacional. Desse amplo processo, prevaleceu a opção por realizar um estudo multicêntrico com a participação dos três níveis de gestão do SUS, apoio de instituições de ensino superior e de entidades profissionais, envolvendo cidades pequenas e grandes, áreas rurais e urbanas, escolas e domicílios, no mais elevado rigor científico. Os dados produzidos deveriam permitir comparações com informações epidemiológicas anteriores e dados internacionais. É do conjunto dessas discussões que decorreu a inclusão de todas as capitais no plano amostral e outras características, como o número de municípios de pequeno porte onde se concentra a menor proporção da população brasileira.

Embora esse desenho possa parecer exagerado para especialistas em amostragem, essa deliberação visou a atender às duas características gerais mencionadas. Dado o caráter multicêntrico, foram elaborados manuais contendo instruções detalhadas para uniformizar os procedimentos de pesquisa, definindo um supervisor para as atividades de campo em cada município. Tratando-se de estrutura complexa de amostragem, os modelos de relatórios foram elaborados visando a permitir a ponderação das estimativas.

Não obstante as dificuldades que marcaram o planejamento e a realização do levantamento, cabe mencionar que estas dificuldades eram esperadas, pois a coleta de dados envolvia 250 municípios nas cinco macrorregiões brasileiras, reconhecidamente heterogêneas. Essa tarefa só pôde ser cumprida graças ao envolvimento na coleta dos dados de profissionais ligados ao atendimento em saúde nas instituições públicas. Entretanto, muitos desses profissionais, embora tivessem alguma experiência com esse tipo de investigação, não eram especializados para a realização de pesquisas. Nesse sentido, a capacitação desses profissionais para a coleta de dados 
constituiu um importante processo de troca de experiências entre academia e serviços. Sabe-se que desse processo, por vezes, pode resultar perda de informações cujo registro estava previsto no plano da pesquisa (locais onde foram realizados exames e entrevistas). Contudo, a opção operacional de integrar as unidades do serviço público de saúde no levantamento não decorreu de improvisação, amadorismo, ou falta de opção. Foi, ao contrário, deliberada. Além de atingir os objetivos imediatos da investigação, o SB Brasil 2003 tinha expressamente como objetivo propiciar a articulação de diferentes agências e unidades de saúde, contribuindo na perspectiva da estruturação de um sistema nacional de vigilância em saúde bucal. Para esse fim, era crucial envolver em todas as etapas da pesquisa, notadamente na coleta de dados, recursos humanos das unidades de saúde, contribuindo para que as equipes locais se apropriassem da tecnologia e instrumentos epidemiológicos empregados. Não se tratava apenas de ir a campo e organizadamente colher os dados, mas de viabilizar que o processo fosse apropriado pelos participantes da coleta de dados e do planejamento operacional loco-regional. É preciso enfrentar e superar o desafio de desenvolver a competência brasileira nesse tipo de investigação e, ao mesmo tempo, inseri-la nas práticas de vigilância em saúde. Esse aspecto não foi devidamente considerado na crítica contida no artigo sob análise, em que o SB Brasil 2003 foi visto como um evento de intervenção "purista" de pesquisa e não como processo vinculado a uma estratégia de implementação de práticas de vigilância em saúde bucal, comportando novos estudos, tanto transversais como longitudinais. Tendo vivenciado o processo de coleta dos dados do SB Brasil 2003, parece-nos relevante registrar graus diferenciados de defasagem na formação e experiência dos trabalhadores da saúde envolvidos no processo, fato que, se por um lado explica, pelo menos em parte, as dificuldades referidas para a conclusão, em todo o país, das etapas previstas no projeto, por outro, mostra a necessidade de iniciativas tão ou mais abrangentes como esta, para catalisar a diversidade de interesses e mobilizar os recursos existentes no país a fim de implementar essas práticas nos sistemas locais de saúde, e assim propiciar treinamento e padronização de procedimentos mais adequados.

Ainda que o delineamento deva ser objeto de aprimoramento - nisto todos estamos de acordo - e que, a despeito da participação de especialistas (oriundos de diferentes áreas, inclusive da Estatística) no plano amostral, não tenham sido apontados com o rigor devido todos os estratos, a hipótese central, aventada no artigo sob análise, é que as estimativas não seriam válidas para a população, aspecto que é discutido a seguir.

\section{Resultados do SB Brasil 2003 e} validade das inferências

Segundo Queiroz et al. ${ }^{1}$ (p. 48), "faltou o cálculo dos pesos amostrais necessários para a produção de estimativas válidas para a população brasileira". Isso significa dizer que informações geradas por inquéritos nacionais que fizeram uso de amostras complexas sem o cálculo de pesos amostrais produziram estimativas que não são válidas para a população brasileira. Como o número de inquéritos nacionais com pesos calculados e disponíveis é muito pequeno até recentemente (anos 1990), desse raciocínio estritamente estatístico, decorreria que todos os conhecimentos assim gerados não seriam válidos.

Esse assunto tem recebido maior atenção nas últimas duas décadas, a partir do desenvolvimento de recursos que facilitaram a incorporação dos aspectos que definem um plano amostral complexo na descrição e análise dos dados científicos 7 .

Ora, a validade de qualquer estudo epidemiológico é algo mais complexo que a concepção estritamente estatística advogada pelos autores. Reichenheim \& Moraes ${ }^{8}$ propuseram seis pilares para a apreciação da validade de estudos epidemiológicos, mostrando que o tema engloba, mas não se reduz aos critérios estatísticos da amostragem. Embora tenham tomado estudos analíticos como base de suas observações, muitas das considerações apresentadas a seguir têm por base esse importante artigo publicado em nosso meio, pois a crítica extrapolou para estudos de associação que usam as informações do SB Brasil 2003.

A validade de estudos epidemiológicos é tema razoavelmente complexo, encerra questões não apenas científicas mas também filosóficas, relacionadas à Lógica, Ontologia e Epistemologia. Greenland 9 também assinalou que a validade não se reduz aos aspectos estatísticos, sem dúvida relevantes, mas envolve questões conceituais, operacionais, de modelo de análise, produção das informações e mensuração que devem ser igualmente consideradas.

A inferência de parâmetros populacionais a partir do SB Brasil 2003 exige procedimentos metodológicos específicos às análises de interesse, $\mathrm{o}$ que é complicado pelo grande número de variáveis de diferentes tipos. Com efeito, o conjunto de variáveis relativas à cárie dentária difere bastante das variáveis de acesso a tratamento odontológico e autopercepção de saúde bucal. O artigo não faz essa distinção, mas generaliza as objeções à 
representatividade de todas as estimativas, concluindo indistintamente pela não-validade das inferências. A afirmação genérica e abrangente de que "estimativas válidas para a população brasileira" só são possíveis mediante "o cálculo dos pesos amostrais" é assunto controverso e merece cautela. Os próprios autores mencionam que "para variáveis com reduzida variação na amostra as estatísticas amostrais, embora enviesadas, não serão muito diferentes das estimativas obtidas de forma correta; esse é possivelmente o caso da variável cárie dentária, de elevada prevalência na maioria dos grupos estudados" 1 (p. 49). Embora não fique claro o que os autores entendem como "elevada prevalência" de cárie, argumenta-se que, nesse exemplo, o que possibilita inferência válida para as macrorregiões e o país é o conhecimento existente sobre os valores dos índices ceo-d e CPO-D. Os graus de homogeneidade e heterogeneidade na distribuição da experiência de cárie (índices ceo-d e CPO-D) intra e intermacrorregiões devem ser devidamente considerados para aferir o quanto a aplicação de pesos amostrais a essas variáveis é imprescindível ou não, dependendo do objetivo e desenho de cada estudo. Em nosso entender, esse conhecimento sobre a epidemiologia da cárie é essencial e não deve ser desconsiderado ao se analisar o significado e os efeitos do enviesamento.

Quanto à "falta dos pesos amostrais" inviabilizar "a estimação de totais populacionais”, isto seria uma limitação para estudos e análises em que estes totais populacionais fossem imprescindíveis. Mas isso não significa a interdição de inúmeras possibilidades de estudos relevantes, tematizando diferentes aspectos da base de dados, que se transformaram em publicações em revistas científicas, no Brasil e no exterior, e em dezenas de trabalhos apresentados em congressos. A Tabela 1 sintetiza algumas medidas comparando resultados de descrição e análise do SB Brasil 2003 com e sem ponderação da amostra, para variáveis com diferentes níveis de prevalência. Como não se dispõe de informações completas para realizar a ponderação da amostra, esses resultados não constituem correções definitivas das estimativas, correspondem apenas a um ensaio de ponderação, realizado para indicar a magnitude das diferenças obtidas em medidas de frequência e associação entre variáveis. Para seu cálculo, empregou-se o próprio esquema de pesos indicado por Queiroz et al. 1. Os resultados desse ensaio indicam que o impacto da atribuição de pesos de amostragem sobre as medidas de prevalência pode ser reduzido, pouco relevante, como já havia sido relatado para outro banco de dados ${ }^{10}$. Quanto às medidas de associação, o efeito da correção seria ainda menor, correspondendo a alterações em casas decimais, sem impacto em magnitude e sentido das interpretações analíticas. Assim, não seria correto colocar em dúvida o valor científico desses artigos e a

Tabela 1

Comparação entre estimativas com e sem ponderação amostral: prevalência de condições de saúde bucal e razão de prevalências avaliando a associação entre cárie não tratada e renda familiar. Adolescentes (15-19 anos), SB Brasil 2003.

\begin{tabular}{|c|c|c|c|c|}
\hline \multirow[t]{2}{*}{ Variáveis } & \multicolumn{2}{|c|}{ Sem ponderação amostral * } & \multicolumn{2}{|c|}{ Com ponderação amostral ** } \\
\hline & Prevalência (\%) & $\mathrm{IC} 95 \%$ & Prevalência (\%) & IC95\% \\
\hline Bolsa periodontal & 1,3 & $1,0-1,7$ & 1,5 & $0,9-2,0$ \\
\hline Necessidade de tratamento endodôntico & 12,4 & $11,2-13,6$ & 12,2 & $10,9-13,6$ \\
\hline Dor de dente & 43,8 & $37,7-50,0$ & 45,4 & $36,0-53,9$ \\
\hline Cárie não tratada & 65,8 & $63,6-67,9$ & 60,6 & $58,0-63,2$ \\
\hline \multirow[t]{2}{*}{ Associação entre cárie não tratada e renda familiar } & \multicolumn{2}{|c|}{ Sem ponderação amostral } & \multicolumn{2}{|c|}{ Com ponderação amostral } \\
\hline & RP & IC95\% & RP & IC95\% \\
\hline Menos de 1 salário mínino & 1,00 & Referência & 1,00 & Referência \\
\hline 1 a 2 salários míninos & 0,98 & $0,93-1,03$ & 1,02 & $0,95-1,08$ \\
\hline 2 a 4 salários míninos & 0,88 & $0,83-0,93$ & 0,90 & $0,82-0,98$ \\
\hline 4 ou mais salários míninos & 0,68 & $0,63-0,74$ & 0,70 & $0,63-0,79$ \\
\hline Renda ignorada & 0,92 & $0,82-1,05$ & 0,88 & $0,75-1,02$ \\
\hline
\end{tabular}

IC95\%: intervalo de 95\% de confiança; RP: razão de prevalências.

* Não foram utilizados pesos, mas foi considerado o desenho complexo da amostra;

** A ponderação utilizou esquema de pesos indicado por Queiroz et al. 1. 
correção dessas análises, como pode-se, inadvertidamente, denotar do artigo sob exame, a partir de afirmações genéricas e generalizantes sobre "validade" das inferências.

Pode-se arguir que a análise não é eficiente, posto que os intervalos de confiança podem ser mais amplos do que seria desejável e, portanto, com menor poder estatístico para rejeitar a hipótese de nulidade 11,12. Entretanto, não convém fazer esse julgamento por atacado, cada estudo deve ser avaliado considerando, entre outros aspectos, os recursos analíticos adotados para neutralizar os efeitos gerados pela ausência de estimativas ponderadas e os níveis de precisão assumidos pelos pesquisadores.

A estatística deve ser ferramenta para a análise epidemiológica, não deveria se arrojar como dirigente desses estudos, tentando determinar o que pode e não pode ser feito em epidemiologia. Isso é tanto mais relevante quando se constata que, desde 2003 até o início de 2009, foram publicados 27 artigos em periódicos científicos bem qualificados nacional e internacionalmente, os quais utilizaram a base de dados do SB Brasil para estudar diferentes questões de saúde bucal e produzir conhecimento relevante para o planejamento em saúde. Esses trabalhos investigaram, entre outros aspectos, fatores associados à experiência de cárie $13,14,15$, perda dentária e edentulismo 16,17,18, dor nos dentes e gengivas 19,20, condições gengivais e doença periodontal 21,22, autopercepção de saúde bucal 23,24, acesso a serviços odontológicos 25,26 , necessidades de tratamento 27,28,29, acesso à água fluoretada 30,31. Na comparação com resultados de levantamentos anteriores, foi possível reconhecer e examinar o processo de declínio e polarização da prevalência de cárie no Brasil. Também foram descritas e analisadas desigualdades no acesso a tratamento odontológico e aspectos metodológicos importantes como o treinamento e calibração dos examinadores. O número de publicações científicas analisando dados do SB Brasil 2003 é expressivamente maior que o número resultante dos módulos de saúde da Pesquisa Nacional por Amostra de Domicílios de 1998 (PNAD 1998) e da Pesquisa Mundial de Saúde no Brasil, para mencionar apenas dois amplos inquéritos recentes, sobre os quais também incidem alguns aspectos criticados na publicação examinada neste estudo, como a ausência de uma variável no banco de dados da PNAD 1998 que permitisse ponderação de estimativas.

Essas publicações justificam plenamente, a nosso ver, "o custo da pesquisa" e são de incalculável valor "para o planejamento de saúde bucal no país", justamente ao contrário do que se afirma no artigo sob análise, em que se condiciona a justificativa da realização da pesquisa ao "cálculo dos pesos amostrais". Apenas para ilustrar, veja-se a ampliação dos investimentos federais nesta área, orientados ao enfrentamento das iniquidades em saúde bucal, com base em achados do SB Brasil 2003, favorecendo a população mais vulnerável que pode se beneficiar por ter suas condições mais bem conhecidas, como no caso do edentulismo e das próteses dentais. Além disso, esses estudos confirmaram menores níveis de cárie em regiões com fluoretação de águas, mostrando a atualidade e importância desta medida de saúde pública.

Os registros remetendo à noção de "representatividade" da população brasileira, nos dados do SB Brasil 2003, aparecem em sete contextos no artigo. O conceito de representatividade subjacente vincula essa noção aos critérios estatísticos de estimativa e de inferência. Nesse plano, não há o que objetar. O problema é que para certos fins epidemiológicos, a depender das variáveis em questão e da finalidade da análise na qual emerge a questão da representatividade, o critério estatístico não é o único a ser considerado e, na impossibilidade de utilizá-lo, outros podem ser empregados. Além disso, mesmo do ponto de vista estatístico, os requerimentos para o uso de técnicas mais ou menos exigentes do ponto de vista operacional dependem da finalidade e da possibilidade de obtenção de dados.

O objetivo de muitos estudos epidemiológicos de base populacional é gerar uma espécie de "fotografia" da situação. Nos manuais de epidemiologia é recorrente o termo "fotografia" para explicar o conceito de prevalência. É compreensível, pois na maioria das vezes, tudo o que se busca é obter uma "imagem da situação" relativa ao problema epidemiológico de interesse. O grau de nitidez, a precisão exigida dessa imagem depende da finalidade de cada estudo.

Mas, até que ponto uma imagem é "representativa", tendo em vista sua capacidade de captar o que importa, de revelar o que se busca conhecer? Em termos estatísticos, tudo depende dos números: dados sobre a população de referência, tamanho da amostra, probabilidade de cada indivíduo da população pertencer à amostra, dentre outros aspectos. Feitos os cálculos, concluise sobre a representatividade das estimativas e validade das inferências. Mas, o que concluir quando a imagem obtida não permitir "estimar parâmetros populacionais" porque não se pode assegurar que a amostra não é enviesada? Poderse-ia falar em "representatividade" de "imagens" assim obtidas? Epidemiologistas ortodoxos responderiam que não. Outros, epidemiologistas ou não, podem responder que depende e que, talvez, sim. É o nosso caso. Compartilhamos o 
entendimento de que o fato de não se poder afirmar que valores obtidos de uma amostra equivalem aos seus parâmetros populacionais não autoriza afirmar que estes valores não equivalem aos seus respectivos parâmetros, pois "pode ser" que sejam equivalentes. Afirmar, categoricamente, que não são equivalentes, como os autores fizeram, requer demonstração baseando-se em evidência empírica. Esse requisito decorre de lógica elementar: em muitas situações, conhecimentos (valores) obtidos por meios não estatísticos, coincidem com os obtidos utilizando-se esses meios. Estimativas populacionais enviesadas podem eventualmente coincidir com estimativas populacionais não-enviesadas. Cabe, a quem afirma que isso não ocorre, demonstrá-lo. A título de ilustração, pode-se utilizar uma imagem cara a professores de estatística: é possível que as árvores da margem da floresta não representem o conjunto de árvores da floresta. Mas também pode ser que representem. Afirmar que as árvores da margem não são representativas do conjunto requer demonstração. Certas amostras, para algumas variáveis e dependendo dos critérios, podem gerar estimativas que correspondem, para o "conhecimento da floresta", a mais, muito mais do que, apenas, as árvores da margem da floresta. Falta, ao artigo de Queiroz et al. 1, essa cautela a respeito de possibilidades, com as quais opera permanentemente a epidemiologia, mas que não se coaduna com requisitos da análise estatística mais ortodoxa.

Ademais, certas imagens, mesmo "válidas" e "representativas", podem prescindir de maior nitidez ou precisão e, ainda assim, corresponder a conhecimento relevante, significativo, útil para determinados fins. Como exemplo, três utilíssimas imagens para conhecer a realidade não costumam ser postas em questão quanto a "representatividades": (i) o quadro Guernica, dePablo Picasso (http://www.museoreinasofia.es/coleccion/ obras/guernica.html), é uma "imagem" que, sem atender a nenhum critério estatístico, representa não apenas o horror do massacre na cidade basca em 1937, na guerra civil espanhola, mas representa o horror de todas as guerras. $\mathrm{O}$ mesmo fato histórico foi pintado por Salvador Dalí, igualmente representativo do espanto e horror. Ambos fazem "inferências válidas" daquela realidade, também captada, em parte, de modo sem dúvida válido pela foto da Figura 1; (ii) o quadro Impressões - O Nascer do Sol (Figura 2), de Claude Monet, símbolo do impressionismo e da ruptura estética desse movimento, corresponde a uma imagem de nascer do sol num porto que pode causar profundo estranhamento pela imprecisão das formas. Não obstante, não há dúvida de que há ali um porto, água, embarcação, sol nascen- do... A imagem é, independentemente do que se pense sobre ela, representativa do que se propôs a revelar; e (iii) a célebre capa que Siron Franco preparou para a revista Veja (edição 1320, de 29 de dezembro de 1993; http://veja.abril.com.br/ arquivo_veja/capa_29121993.shtml), compondo o rosto de Herbert de Souza Filho com um punhado de grãos de cereais de vários tamanhos, formas e cores. Reunidos, distribuídos no espaço e organizados conforme quis o gênio do artista plástico goiano, o rosto de Betinho emerge inconfundível dessa inusitada combinação de milho, arroz, feijão...

Em todos esses casos, fotografias obtidas com os melhores equipamentos talvez gerassem imagens mais precisas e fiéis à realidade. Nem é o caso de questionar o leitor sobre o que preferiria: Picasso, Monet, Siron ou fotografias "de 10 megapixels”. A resposta poderia ser: depende. Depende do momento, da finalidade, de outras circunstâncias. O ponto central nessa reflexão sobre representatividade de estimativas e validade de inferências em epidemiologia é que, para os três exemplos, não sendo possível obter tal imagem de 10 megapixels, mesmo as imagens que tragam a marca da "precariedade" impressionista, da "deformação" cubista e da singeleza de tomates e batatas dispersos organizadamente podem bastar para que o objeto do conhecimento configure imagem útil aos olhos de quem vê.

Esse recurso argumentativo à estética não é despropositado. Muitos inquéritos populacionais nos proporcionam não mais que imagens impressionistas, cubistas, singelas até, das situações que queremos conhecer em termos epidemiológicos. Se uma "imagem de 10 megapixels” é possível, tanto melhor, ou seja: busca-se “estimativas não-enviesadas”. Quando, porém, isso não é factível, há que se dispor de alternativas e fazer aflorar "imagens" úteis, ainda que menos precisas. A questão central é se tais imagens são "válidas" e se o critério estatístico deve ser o único a ser considerado. Entendemos que além do estatístico, outros critérios devem ser levados em conta. Refletindo sobre o mecanicismo que contrai os conhecimentos biológicos às leis da Física e Química, Hempel 32 nos alerta sobre os problemas da redução teórica nas ciências naturais. Tomando esse ensaio como advertência cabível, ainda que com ressalvas, à prática da pesquisa epidemiológica, há que perguntar se o uso da Estatística como único critério não corresponde a reducionismo teórico que, do ponto de vista prático, serve apenas para manter a separação entre o mundo dos serviços e o mundo acadêmico, conforme mencionado por Castiel \& Rivera 33, a propósito do divórcio entre epidemiologia e planejamento em saúde. 


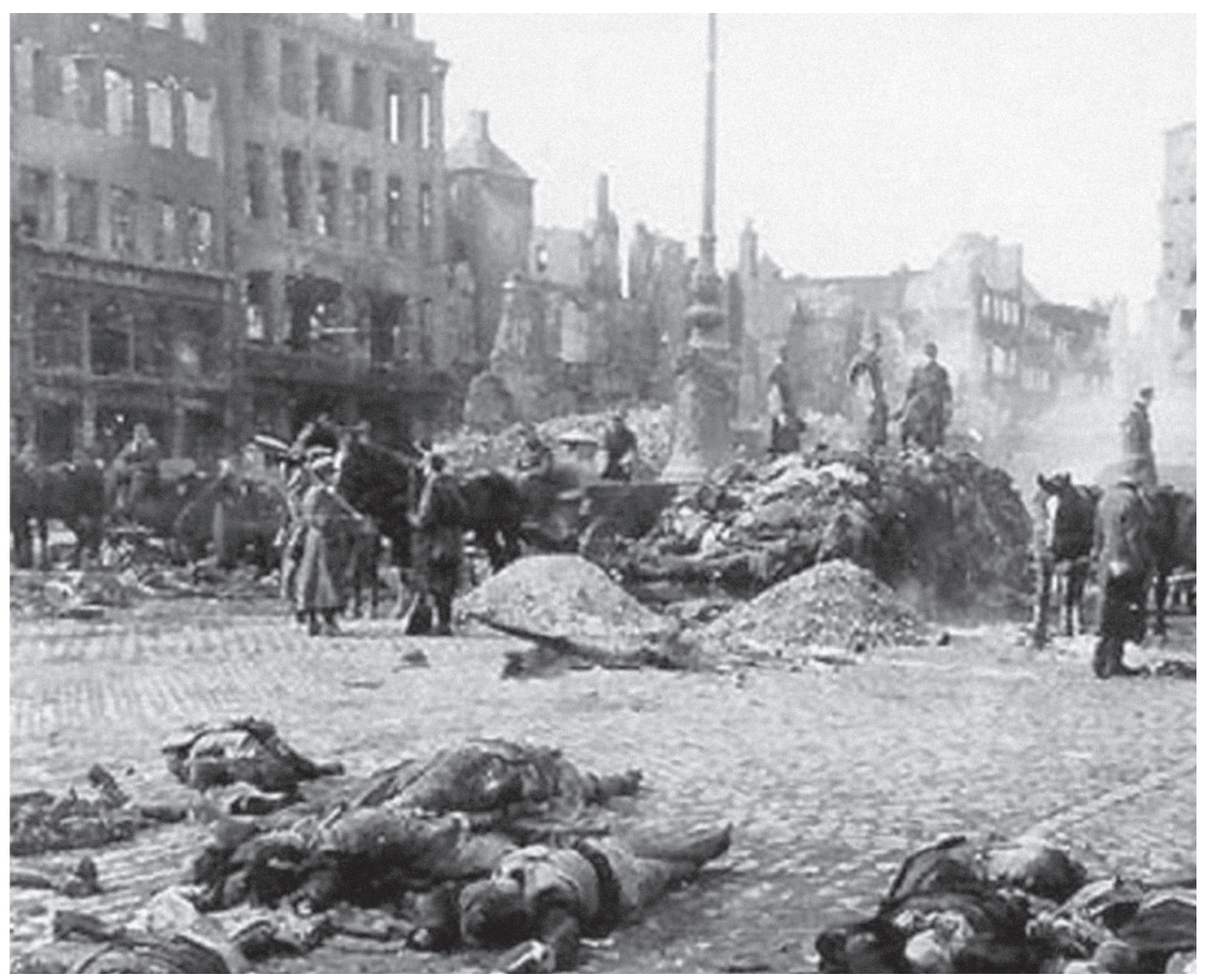

É preciso cautela com relação ao modo como a ciência opera para conhecer, para desvelar a realidade. O conhecimento científico é "um" discurso, não "o" discurso sobre a realidade. Assinale-se, ainda, que o conhecimento obtido com "amostras não-enviesadas” será sempre, enquanto discurso produzido a partir de uma (dentre outras possíveis) aproximação à realidade, mediado por limites e restrições inerentes ao saber científico. Em outras palavras: potencialmente também conterá falhas. Sempre será possível arguir a validade de qualquer inferência, ainda que produzida segundo a melhor técnica estatística. O que está em questão, nesse aspecto, é a reconhecida limitação da ciência em, sendo um discurso sobre a realidade, reproduzi-la, apreendê-la, capturá-la. Não obstante, o tom adotado no artigo sob análise não relativiza nunca: é tom de quem julga e desqualifica, pois se vê como portador de uma verdade científica inquestionável. As estimativas não são representativas, pois não resultam de inferências válidas e ponto final.

\section{Pesquisadores, gestores e metas programáticas}

A ideia central que atravessa todo o texto é de que "as estimativas do SB Brasil 2003 não são válidas para a população". Queiroz et al., crêem ser necessário produzir "inferências válidas", pois seria isto que, "em última instância", justificaria "o custo de qualquer pesquisa" e, por conseguinte, os custos decorrentes da realização do SB Brasil 2003. Mais: somente após produzir as "inferências válidas para a população", seria possível aos pesquisadores e gestores "analisar as reais [sic] condições de saúde bucal no país e traçar metas para seu melhoramento" 1 (p. 57). 


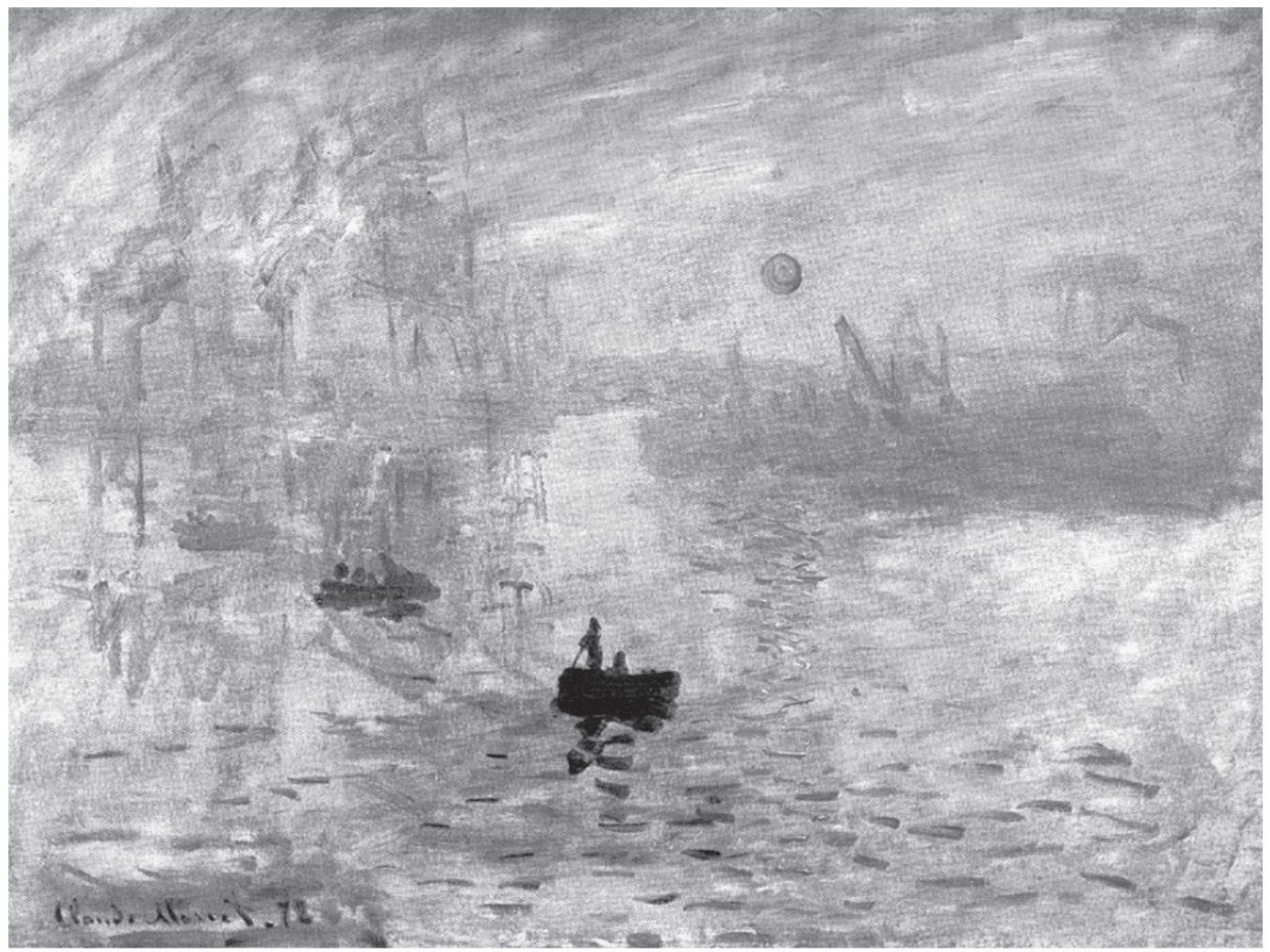

Essa ideia é exagerada, não corresponde à realidade. O país tem uma política nacional de saúde bucal reconhecidamente bem-sucedida e pode perfeitamente fixar metas com razoável grau de precisão. Mesmo admitindo a hipótese de que isso seria possível apenas com o emprego de pesos amostrais para estimar parâmetros populacionais para o SB Brasil 2003, é também possível admitir a hipótese de que os dados disponíveis permitem conhecer relativamente bem o que se passa com a saúde bucal dos brasileiros. Vimos que vários estudos foram feitos com base nos dados desse banco, produzindo conhecimento relevante, apesar da ausência de pesos amostrais. A aparente interdição que se pretende fazer ao emprego desses dados não se justifica. Ao contrário, os resultados desses estudos têm contribuído de modo importante para subsidiar decisões no âmbito do planejamento em saúde bucal. Para isso, pode ser que os gestores venham se utilizando de imagens não plenamente níti- das, que corresponderiam a algo como um impreciso quadro impressionista, ou cubista. Mas o fazem com a consciência e a generosidade de quem reconhece o conteúdo, as possibilidades e os limites de imagens como as proporcionadas por Monet ou Picasso. O requisito indispensável para isso é saber ver e reconhecer o que é e o que não é representativo e válido. Em certas situações é preciso muito mais do que estatística e lógica formal. Goethe 34 escreveu, em outro contexto, que "cinza é toda teoria, mas verde é a árvore da vida”. Essa reflexão, citada por muitos que buscam aliar pensamento e ação, decorre do reconhecimento, nessa metáfora de cores, que entre cinza e verde, com todas as urgências, contradições, limites e possibilidades, a vida é sempre muito mais rica e complexa do que qualquer teoria. Essa metáfora tem, também, implicações epistemológicas, relacionadas à possibilidade do conhecimento científico apreender a riqueza e complexidade da "verde árvore da vida", e ao 
modo de como fazê-lo dispondo, muitas vezes, apenas dos cinzentos instrumentos da teoria. Outro poeta, o português Fernando Pessoa, recuperou o adágio dos argonautas de que "navegar é preciso; viver não é preciso" 35. A vida, pode-se depreender, não segue em retas e curvas exatas, como as requeridas para os planos de navegação. O navegar deve ser exato, preciso, mas o viver não é assim. Há imprecisão no viver. É com essa inexatidão do viver que lidam os que trabalham com saúde, os que têm de aliar pensamento e ação em processos que envolvem dezenas, centenas, milhares de pessoas em projetos institucionais, de alcance coletivo, como foi o empreendimento do SB Brasil 2003. A imprecisão das imagens que se podem obter valendo-se dessa base de dados não deve corresponder ao não reconhecimento do seu valor. Ao contrário, o SB Brasil 2003 deve ser visto e analisado a partir desse quadro mais geral em que está inserido, de valorização e desenvolvimento de práticas de vigilância da saúde bucal coletiva, como momento de um processo e não como um fato isolado, e que procuramos detalhar neste artigo. Alguns de seus dados podem não ser precisos, mas são necessários. E úteis, pois têm valor inestimável.

\section{Resumo}

Problematiza-se a afirmação de que não são válidas as estimativas sobre as condições de saúde bucal da população brasileira geradas pelo SB Brasil 2003. Criticam-se os elementos que pretendem sustentar esse ponto de vista com base apenas em conceitos estatísticos, sem prova empírica. Identificam-se reduções decorrentes da abordagem epistemocêntrica que recusa peremptoriamente outras formas de conhecimento e não reconhece o caráter multidisciplinar da epidemiologia. Reconstituem-se informações sobre a realização do levantamento e seu impacto na produção de conhecimento. Faz-se uma analogia entre ciência e arte, argumentando-se que, nas imagens obtidas por ambas, os saberes gerados a partir do objeto cognoscivel assumem feições variadas e, portanto, o reconhecimento de sua validade requer amplo domínio do objeto e operações com adequados critérios de valor. Conclui-se pela cientificidade, validade e relevância da produção acadêmica desenvolvida a partir da base de dados do levantamento SB Brasil 2003.

Aplicações da Epidemiologia; Levantamentos de Saúde Bucal; Saúde Bucal

\section{Colaboradores}

P. C. Narvai elaborou o esboço inicial, coordenou a equipe do estudo e colaborou para a redação da versão final. J. L. F. Antunes elaborou o esboço inicial, realizou o ensaio de ponderação da amostra e colaborou para a redação da versão final. S. J. Moysés, P. Frazão, M. A. Peres, K. G. Peres, M. L. R. Sousa e A. G. Roncalli participaram de todas as etapas de discussão e colaboraram para a redação da versão final.

\section{Referências}

1. Queiroz RCS, Portela MC, Vasconcellos MTL. Pesquisa sobre as Condições de Saúde Bucal da População Brasileira (SB Brasil 2003): seus dados não produzem estimativas populacionais, mas há possibilidade de correção. Cad Saúde Pública 2009; 25:47-58.

2. Departamento de Atenção Básica, Secretaria de Atenção à Saúde, Ministério da Saúde. Projeto SB Brasil 2003. Condições de saúde bucal da população brasileira, 2002-2003: resultados principais. Brasília: Ministério da Saúde; 2004.

3. Bardin L. Análise de conteúdo. Lisboa: Edições70/ São Paulo: Editora Martins Fontes; 2002.

4. Divisão Nacional de Saúde Bucal, Ministério da Saúde. Levantamento epidemiológico em saúde bucal: Brasil, zona urbana. Brasília: Ministério da Saúde; 1988.

5. Departamento de Atenção Básica, Secretaria de Atenção à Saúde, Ministério da Saúde. Levantamento epidemiológico em saúde bucal: 1a etapa - cárie dental. Brasília: Ministério da Saúde; 1996.

6. Barata RB. Epidemiologia social. Rev Bras Epidemiol 2005; 8:7-17.

7. Silva PLN, Pessoa DGC, Lila MF. Análise estatística de dados daPNAD: incorporando a estrutura do plano amostral. Ciênc Saúde Coletiva 2002; 7:659-70.

8. Reichenheim ME, Moraes CL. Alguns pilares para a apreciação da validade de estudos epidemiológicos. Rev Bras Epidemiol 1998; 1:131-48.

9. Greenland S. Concepts of validity in epidemiological research. In: Detels R, McEwen J, Beaglehole $\mathrm{R}$, Tanaka H, editors. Oxford textbook of public health. v. 2: the methods of public health. $4^{\text {th }} \mathrm{Ed}$ New York: Oxford University Press; 2001. p. 621-39. 
10. Sousa MH, Silva NN. Estimativas obtidas de um levantamento complexo. Rev Saúde Pública 2003; 37:662-70.

11. Kom EL, Graubard BI. Epidemiologic studies utilizing surveys: accounting for the sampling design. Am J Public Health 1991; 81:1166-73.

12. Szwarcwald CL, Damacena GN. Amostras complexas em inquéritos populacionais: planejamento e implicações na análise estatística dos dados. Rev Bras Epidemiol 2008; 11 Suppl 1:38-45.

13. Narvai P, Frazão P, Roncalli A, Antunes J. Cárie dentária no Brasil: declínio, polarização, iniqüidade e exclusão social. Rev Panam Salud Pública 2006; 19:385-93

14. Antunes JLF, Jahn GM, Camargo MA. Increasing inequalities in the distribution of dental caries in the Brazilian context. Community Dent Health 2005; 22:94-100.

15. Antunes JLF, Peres MA, Mello TRC, Waldman EA. Multilevel assessment of determinants of dental caries experience in Brazil. Community Dent Oral Epidemiol 2006; 34:146-52.

16. Rihs LB, Silva DD, Sousa MLR. Dental caries in an elderly population in Brazil. J Appl Oral Sci 2009; 17:8-12.

17. Barbato PR, Peres MA. Tooth loss and associated factors in adolescents: a Brazilian populationbased oral health survey. Rev Saúde Pública 2009; 43:13-25.

18. Hugo FN, Hilgert JB, Sousa MLR, Silva DD, Pucca Jr. GA. Correlates of partial tooth loss and edentulism in the Brazilian elderly. Community Dent Oral Epidemiol 2007; 35:224-32.

19. Antunes JLF, Peres MA, Frias AC, Crosato EM, Biazevic MGH. Saúde gengival de adolescentes e a utilização de serviços odontológicos, Estado de São Paulo. Rev Saúde Pública 2008; 42:191-9.

20. Borges CM, Cascaes AM, Fischer TK, Boing AF, Peres MA, Peres KG. Dor nos dentes e gengivas e fatores associados em adolescentes brasileiros: análise do inquérito nacional de saúde bucal SB-Brasil 2002-2003. Cad Saúde Pública 2008; 24:1825-34.

21. Cascaes AM, Peres KG, Peres MA. Periodontal disease is associated with poor self-rated oral health among Brazilian adults. J Clin Periodontol 2008; 36:25-33.

22. Peres MA, Antunes JLF, Boing AF, Peres KG, Bastos JLD. Skin colour is associated with periodontal disease in Brazilian adults: a population-based ora health survey. J Clin Periodontol 2007; 34:196-201.
23. Martins AMEBL, Barreto SM, Pordeus IA. Autoavaliação de saúde bucal em idosos: análise com base em modelo multidimensional. Cad Saúde Pública 2009; 25:421-35.

24. Matos DL, Lima-Costa MF. Auto-avaliação da saúde bucal entre adultos e idosos residentes na Região Sudeste: resultados do Projeto SB-Brasil, 2003. Cad Saúde Pública 2006; 22:1699-707.

25. Antunes JLF, Peres MA, Jahn GM, Levy BB. The use of dental care facilities and oral health: a multilevel approach of schoolchildren in the Brazilian context. Oral Health Prev Dent 2006; 4:287-94.

26. Barbato PR, Nagano HCM, Zanchet FN, Boing AF, Peres MA. Perdas dentárias e fatores sociais, demográficos e de serviços associados em adultos brasileiros: uma análise dos dados do Estudo Epidemiológico Nacional (Projeto SB Brasil 20022003). Cad Saúde Pública 2007; 23:1803-14.

27. Antunes JLF, Peres MA, Mello TRC. Determinantes individuais e contextuais da necessidade de tratamento odontológico na dentição decídua no Brasil. Ciênc Saúde Coletiva 2006; 11:79-87.

28. Frias AC, Antunes JLF, Junqueira SR, Narvai PC. Determinantes individuais e contextuais da prevalência de cárie dentária não tratada no Brasil. Rev Panam Salud Pública 2007; 22:279-85.

29. Gushi LL, Rihs LB, Soares MC, Forni TIB, Vieira V, Wada RS, et al. Cárie dentária e necessidades de tratamento em adolescentes do Estado de São Paulo, 1998 e 2002. Rev Saúde Pública 2008; 42:480-6.

30. Gabardo MC, Silva WJ, Olandoski M, Moysés ST, Moysés SJ. Inequalities in public water supply fluoridation in Brazil: an ecological study. BMC Oral Health 2008; 8:9.

31. Peres MA, Antunes JLF, Peres KG. Is water fluoridation effective in reducing inequalities in dental caries distribution in developing countries? Soz Präventivmed 2006; 51:302-10.

32. Hempel CG. Filosofia da ciência natural. Rio de Janeiro: Jorge Zahar Editor; 1981.

33. Castiel LD, Rivera FJU. Planejamento em saúde e epidemiologia no Brasil: casamento ou divórcio? Cad Saúde Pública 1985; 1:447-56.

34. Goethe JW. Fausto. 2a Ed. Aveiro: Livraria Clássica Editora; 1919.

35. Pessoa F. Livro do desassossego. Lisboa: Editora Ática; 1982.

Recebido em 27/Mar/2009

Versão final reapresentada em 02/Out/2009

Aprovado em 10/Dez/2009 